\title{
Formation of the third bodies of steel sliding against brass under lubricated conditions
}

\author{
Lin Liu a, e, *, Yuanyuan Sheng a, e, Ming Liu ${ }^{\text {a, e }}$, Martin Dienwiebel b, c *, \\ Zhichen Zhang a, e, Davoud Dastan ${ }^{\mathrm{d}}$ \\ a School of Mechanical Engineering, Changzhou University, 213164 Changzhou, Jiangsu, China \\ b Institute for Applied Materials, Karlsruhe Institute for Technology, 76128 Karlsruhe, Germany \\ c Fraunhofer Institute for Mechanics of Materials IWM, 79108 Freiburg, Germany \\ d School of Materials Science and Engineering, Georgia Institute of Technology, 30332 Atlanta, \\ Georgia, USA \\ e Jiangsu Key Laboratory of Green Process Equipment, Changzhou University, 213164 Changzhou, \\ Jiangsu, China
}

\section{Highlights}

- A tribometer based on on-line and RNT techniques performs sliding test.

- The formation mechanism of the third bodies subsurface zone is analyzed.

- MML is formed with dynamic oxidation reactions.

- DRX plays a key role in refinement of nano grains in GRZ.

\begin{abstract}
This work investigates the formation mechanism of the third bodies for brass under lubricated conditions. A tribometer based on on-line and radio-nuclide technique (RNT) techniques is used to perform the sliding experiment of brass versus steel. The microstructure, micro-hardness and chemical composition of the third bodies are analyzed by mean of the scanning electron microscope (SEM), focused ion beam microscope (FIB), transmission electron microscope (TEM), hardness testers and X-ray photoelectron spectroscopy (XPS). The results indicate that the contact pairs show good tribological behaviors and the layered third bodies (mechanically mixed layer (MML), grain refinement zone (GRZ) and plastic deformation zone (PDZ)) are formed on the brass surface during sliding. The formation mechanism of the third bodies is explored. The MML gradually forms on the worn surface in the later stage of the test by proceeding of oxidation reactions. Due to the severe plastic deformation caused by sliding, the interactional dislocation movements and dynamic recrystallization (DRX) lead to the formation of GRZ with nano-scale grains. The PDZ grains lead to the shear deformation by means of friction stress. Moreover, the generation and accumulation of dislocations result in voids in the PDZ.
\end{abstract}

Keywords: The third bodies; Tribological behavior; Microstructure; Brass

\section{Introduction}

* Corresponding author at: School of Mechanical Engineering, Changzhou University, 213164 Changzhou, Jiangsu, China 
Binary brass has been widely used in producing the transmission parts (e.g. bearings, sleeves, etc.) due to its excellent mechanical properties and heat-conductivity [1-4]. The tribological characteristic of working surface plays an essential role in transmission parts in terms of safety, reliability and service life[5-11]. Therefore, the investigation of surface tribological characteristics is important for the application of transmission parts [12, 13].

The formation of the third bodies caused by friction is considerable scientific interest due to the superior wear resistance of copper and its alloys. First studies go back to Schmalz in 1936[14]. Rigney et al.[15, 16] showed that the wear resistance of copper relies on the function of a third body, which is formed by oxides, transfer material and matrix material. Feser et al.[17]showed that that the running-in of binary brass depends on the formation of a grain-refined layer underneath the surface. Descartes and Berthier [18] observed the presence of several zones within the third body in a wheel-rail contact. Similarly, Scherge et al.[19] showed the presence of different zones in steel. B. Yao et al.[20] investigated the microstructure of surface layer for two kinds of $\mathrm{Cu}$ samples after friction tests. The nanostructured mixing layer (NML) and ultra-fine grained DRX layer are formed on the $\mathrm{Cu}$ surfaces. The wear rate of $\mathrm{Cu}$ is determined by the microstructures of NML and DRX layer. Recently, Jerzy Dryzek et al.[21] found the existence of tribo-layer confirmed by the measurement of the increase and attenuation for positron lifetime, moreover, great amount of dislocations and large vacancy clusters are observed in the layer. Other studies [22-24] showed that the fine wear resistance of copper and its alloys is always related to the deformation of continuous and effective the third bodies, such as mechanical mixed layer, super plastic deformation layer, grain refinement layer etc. [25-28]. The characteristics and function of the third body materials have been widely investigated, however, the formation mechanism of subsurface material for many metals and alloys especially brass is not complete so far.

In this work, brass samples that are subjected to sliding against steel under lubricated conditions using an on-line tribometer, are studied in depth with respect to changes in the microstructure, micro-hardness and their chemical compositions. On this basis, the formation mechanism of third body layer and subsurface zones of brass are analyzed in detail.

\section{Experimental details}

\subsection{Sample preparation}

Binary brass (i.e. $79.8 \%$ copper and 20.2\% Zinc), without any metallic lead (Pb), is slid against the $100 \mathrm{Cr} 6$ bearing steel. The brass plates with a size of $150 \times 75 \times 4 \mathrm{~mm}$ are annealed for 45 minutes at $650^{\circ} \mathrm{C}$ and then cooled down at room temperature. Next, citric acid is used to remove unwanted oxide layers of brass formed during the annealing process. For the contact pair, 100Cr6 spheres with diameter $3.0 \mathrm{~mm}$ are ground and polished to acquire a flat surface. The flat surface of pins is in the range of $\Phi 1.54$ to $\Phi 2.25 \mathrm{~mm}$. The measured Vickers hardness of the plates and spheres are $50 \pm$ $3.3 \mathrm{HV}$ and $830 \pm 11.2 \mathrm{HV}$ respectively. The roughness of samples is measured by a white light interferometer (Pl $\mu$ 2300, Sensofar). The average roughness of the plate and pin flat are 15 and 120 nm respectively.

\subsection{Tribological test setup}

An on-line tribometer with an in-situ holographic microscope is used to complete the tribological 
experiment. The wear measurement is conducted on-line by RNT. The tribometer and RNT measuring system are described in details elsewhere [29, 30]. To avoid external environment impacts on the results of experiments, the sliding tests are performed at $25^{\circ} \mathrm{C}$ and a relative humidity of about $50 \%$. Fuchs Petrolub AG provides the PAO-8 base oil (viscosity of $45.8 \mathrm{~mm}^{2} / \mathrm{s}$ at $40{ }^{\circ} \mathrm{C}$ ) and the oil temperature of the whole experiment is maintained at $35^{\circ} \mathrm{C}$ by a Julabo HE-4 pumping system.

In these experiments, the linear reciprocating track is set to a length of $120 \mathrm{~mm}$ and the velocity is $20 \mathrm{~mm} / \mathrm{s}$, whereas the normal loads range from 1.0 to 8.0MPa. Each experiment is repeated three times under the same working conditions to ensure the accuracy of the experimental data. During the experiment, the initial pressure is logged and the images are recorded after each cycle by an in-situ holographic microscope. In addition, the wear rate is measured by the RNT on-line measuring system.

\subsection{Characterization techniques}

For the micro-hardness test, a hardness tester (HM500) is performed at a load of $300 \mathrm{mN}$ for a dwell time of the 20s before and after the experiment. Each value of the micro-hardness test is repeated three times to obtain the average results. An MTS Nano indenter XP is used to measure the Nano indentation. The Chemical composition of brass in depth direction is characterized by an XPS (PHI 5000).

VHX optical microscope (VHX-5000), SEM (JSM-6510), FIB (Zeiss XB 1540) and TEM (JEOL-2100) are used to observe the microstructure of brass. Thin foil samples are cut from the worn layer for TEM observations using mechanical thinning. The samples are further thinned by the twin-jet electro-polisher (TenuPol-5). In order to observe the microstructure of near surface, the surface with wear tracks of TEM sample is bonded face-to-face with the surface of another brass sample without sliding test [31].

\section{Results and discussion}

\subsection{Tribological test}

Fig. 1(a) shows the results of one typical experiment with contact pressure 2.0 MPa. The curves are the friction coefficient (Red line) and wear rate (Blue line) versus cycles and time respectively. Fig. 1(b) presents characteristic topography images, which are obtained from six different cycles of the sliding experiment. There are three typical stages in this test. Initially (within 1900 cycles), both the friction coefficient and the wear rate exhibit a substantially unsteady and fluctuation trend. The initial values of the friction coefficient are low but then increase sharply within the first few cycles to above 0.20 . The wear rate oscillates and rises to $0.10 \mathrm{mg} / \mathrm{h}$ in the first 1800 cycles. Spalling of the surface is also observed from Fig.1 (b-1 and b-2), which illustrates severe fluctuation of friction in this period. In the second stage (from 1900 cycles to 2800 cycles), there is a speedy drop of friction coefficient to approximately 0.10 , while the wear rate maintains a high level of around $0.10 \mathrm{mg} / \mathrm{h}$. Severe adhesive wear of the brass is still observed in Fig.1 (b-3). In the final stage (after 2600 cycles), the friction coefficient remains stable at around 0.12 as the sliding cycles increasing, and meanwhile, the wear rate decreases to $0.05 \mathrm{mg} / \mathrm{h}$ and keeps stable before 5000 cycles and then drops gradually throughout the remainder of the test. In this process, trace amounts of wear debris particles are peeled off with the sliding cycles, and the fresh surface is constantly exposed. Finally, the generating and peeling of wear debris particles reach a dynamic equilibrium. This is in consistent with the topography images as 
shown in Fig.1 (b-4, b-5, and b-6).

\subsection{Microstructure and chemical composition}

As shown in Fig. 2, numerous shallow furrows, which are distributed randomly, are paralleled to the sliding direction. Several worn pits, which are a typical characteristic of adhesive wear, are clearly observed on the worn surfaces. The similar results had been reported by Moshkovich et al. in Ref. [32].

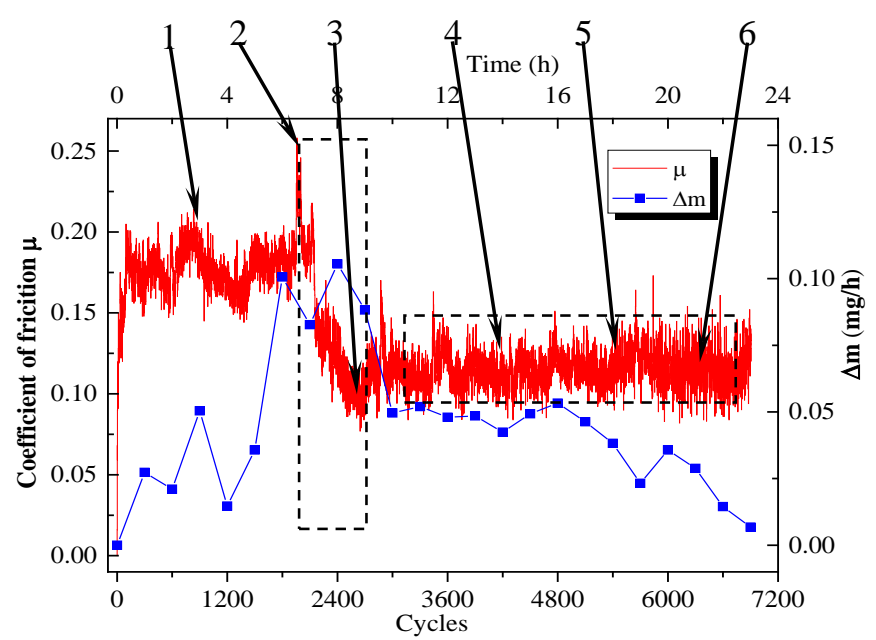

(a)
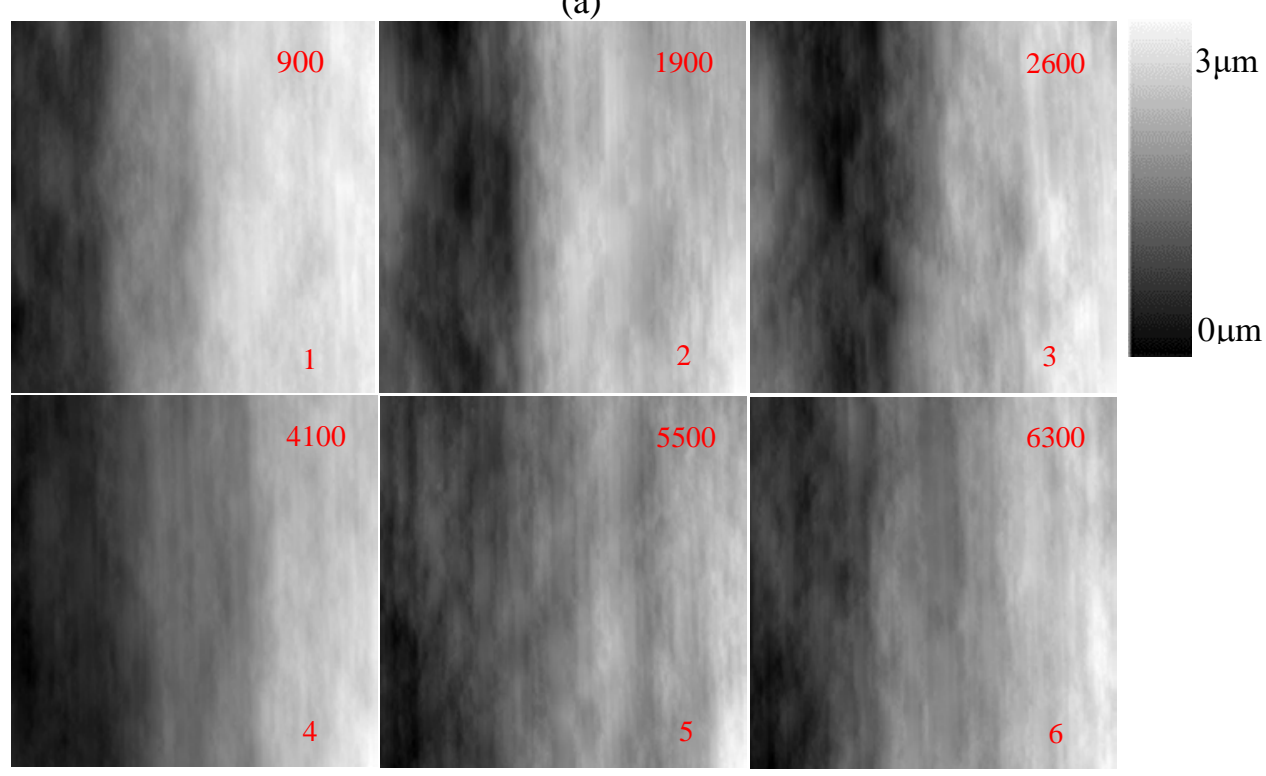

(b)

Fig. 1. Friction coefficient and wear rate vs. cycles and time repectively (a). Characteristic topography images corresponding to specific cycles (b).

The result of the energy dispersive spectrometer (EDS) analysis on the pin surface is shown in Table 1. The worn surface enriches with $\mathrm{Cu}$ (57.71\% wt.), Zn (15.98\% wt.) and O (6.76\% wt.). It is illustrated that the materials transfer from brass to pin and the oxidation reactions occur on the worn surface. Other investigation reported that $\mathrm{ZnO}$ inhibits severe wear and high friction coefficient due to 
difference hardness between two contact pairs [17]. Furthermore, the formation of transfer layer improves wear resistance effectively. This is one of the reasons that the friction coefficient and wear rate are low in the third stage as shown in Fig. 1(a).

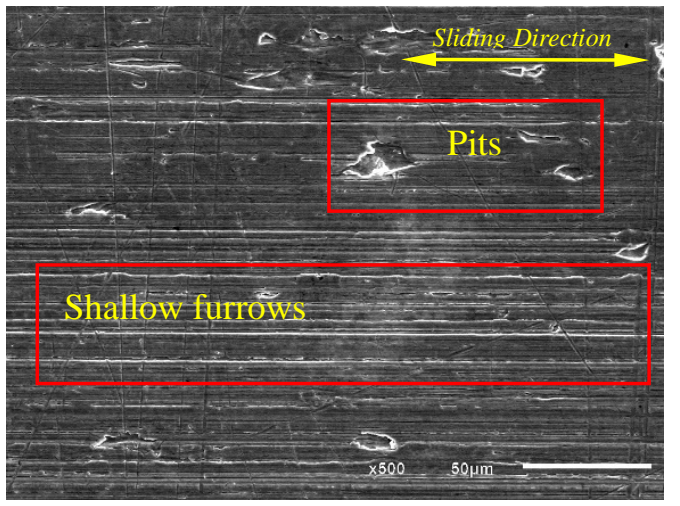

Fig. 2. SEM microstructure of worn surface for brass.

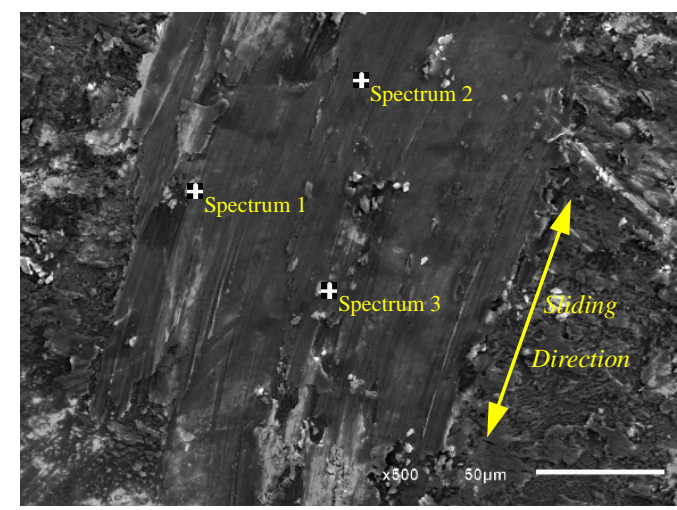

Fig. 3. SEM microstructure of worn surface for pin.

Table 1 Chemical composition of different zones for pin surface

\begin{tabular}{lccccc}
\hline Spectrum & $\mathbf{O K}$ & $\mathbf{C r} K$ & $\mathbf{F e} K$ & $\mathbf{C u} K$ & $\mathbf{Z n} K$ \\
\hline Spectrum1 & 5.76 & 0.56 & 31.66 & 50.76 & 11.26 \\
Spectrum2 & 7.45 & 0.59 & 11.11 & 59.60 & 21.25 \\
Spectrum3 & 7.06 & 0.24 & 11.49 & 65.78 & 15.43 \\
Average & 6.76 & 0.46 & 18.09 & 58.71 & 15.98 \\
Standard deviation & 0.88 & 0.19 & 11.76 & 7.55 & 5.02 \\
Maximum & 7.45 & 0.59 & 31.66 & 65.78 & 21.25 \\
Minimum & 5.76 & 0.24 & 11.11 & 50.76 & 15.43 \\
\hline
\end{tabular}

Fig. 4(a) and 4(b) show the cross-sectional metallographic images of brass before and after test respectively. The grains with the size of approximately $50 \mu \mathrm{m}$ distribute relatively and uniformly as shown in Fig. 4(a). Moreover, a few twinned-grains are shown in the cross-section of brass. Whereas the obvious changes in the cross-section of brass after test are observed in Fig. 4(b). The grains near 
the surface are refined and deformed due to the continual sliding and pressuring between brass and pin. It is indicated that the friction affected layer is formatted during the sliding.

Based on the observations from the brass metallographic structures in Fig. 4(a) and 4(b), the change in the microstructures of brass, from the surface to the depth of about $40 \mu \mathrm{m}$, is obviously due to the sliding test. The microstructure of brass significantly effects on friction and wear properties.

Fig. 5(a) presents the cross-sectional SEM image of brass after sliding. The microstructure near the surface shows an obvious difference with that of the matrix. Obviously, the cross-sectional microstructure of brass is divided into three zones (I, II and III) as shown in Fig. 5(a). The FIB image of brass corresponding to zone (I) of Fig. 5(a) is shown in Fig. 5(b). A large number of voids, which are labeled with rectangular frame in white color, are shown in the upper of the zone (II). Zone (III) is the substrate of brass. In Fig. 5(b), three different zones, which are classified by two dotted lines from the surface to the interior, are defined as the mechanically mixed layer (MML), grain refinement zone (GRZ) and plastic deformation zone (PDZ). The thicknesses of MML and GRZ are about $100 \mathrm{~nm}$ and $3.0 \mu \mathrm{m}$ respectively. No legible grain boundary is observed in MML, while the average grain size in GRZ is approximately $200 \mathrm{~nm}$. The PDZ grains show obviously shear deformation along the sliding direction. The microstructure deformation of surface layer illustrates that the third bodies are formed during the sliding.
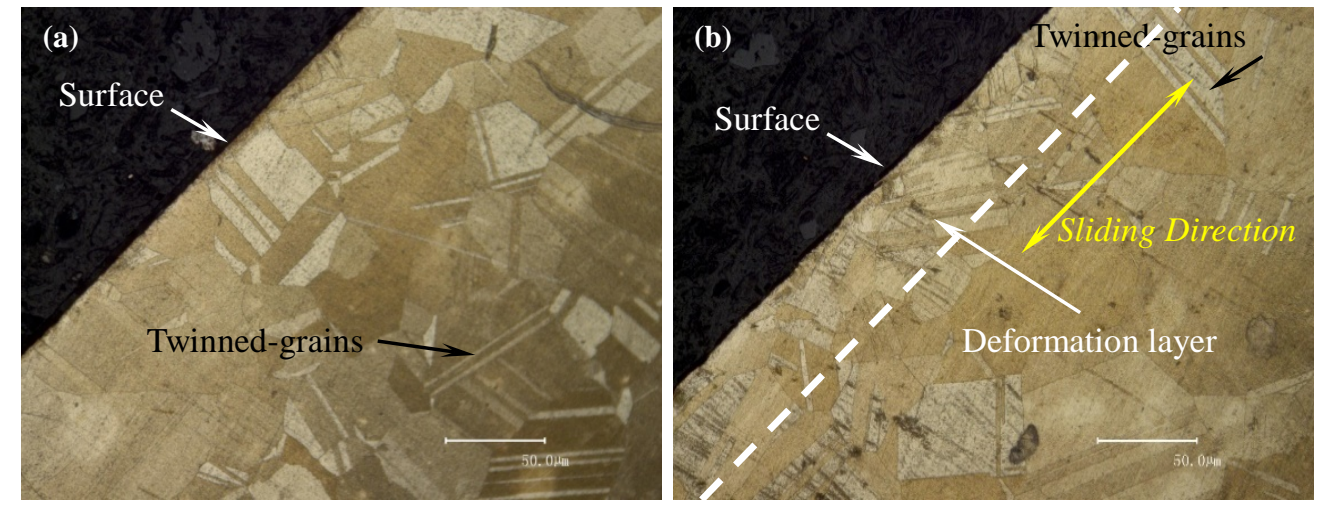

Fig. 4. Cross-sectional metallographic images of brass before (a) and after (b) test.
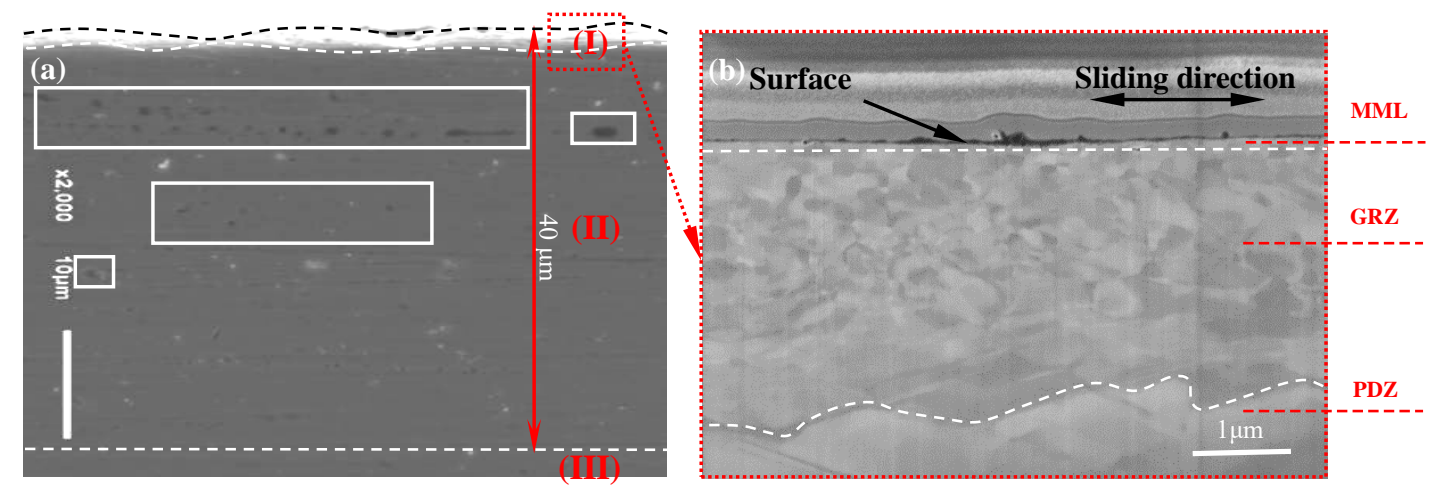

Fig. 5. Cross-sectional SEM image (a) and FIB image (b) of zone (I) in Fig. 5(a). 
TEM images of the microstructures observed in the GRZ at a depth of approximately $2.0 \mu \mathrm{m}$ are shown in Fig. 6. We notice several kinds of dislocation structures, such as dislocation lines (DL) (Fig. 6(a)), dislocation tangles (DT) (Fig. 6(b)), dislocation walls (DW) (Fig. 6(b)), stacking faults (SF) (Fig. 6(c)), etc. Due to the plastic deformation caused by sliding, the friction stress leads to these dislocation structures generated in GRZ. Moreover, the dislocation structures, which are accumulated by continuing sliding, contribute to the refinement of nano-grains in Fig. 6(d) (twinned-grains (TG)).

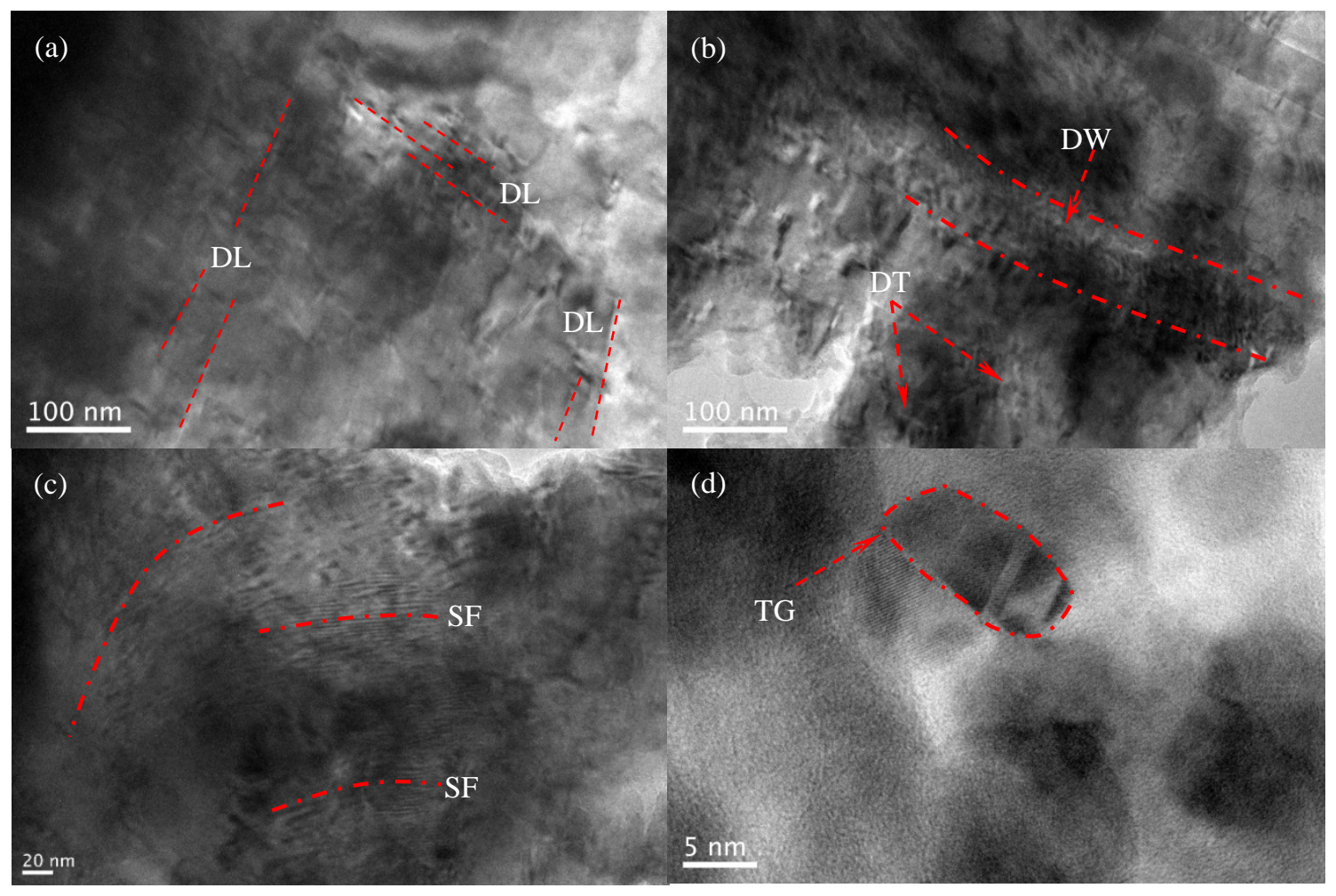

Fig. 6. TEM images of worn surface layer for brass 


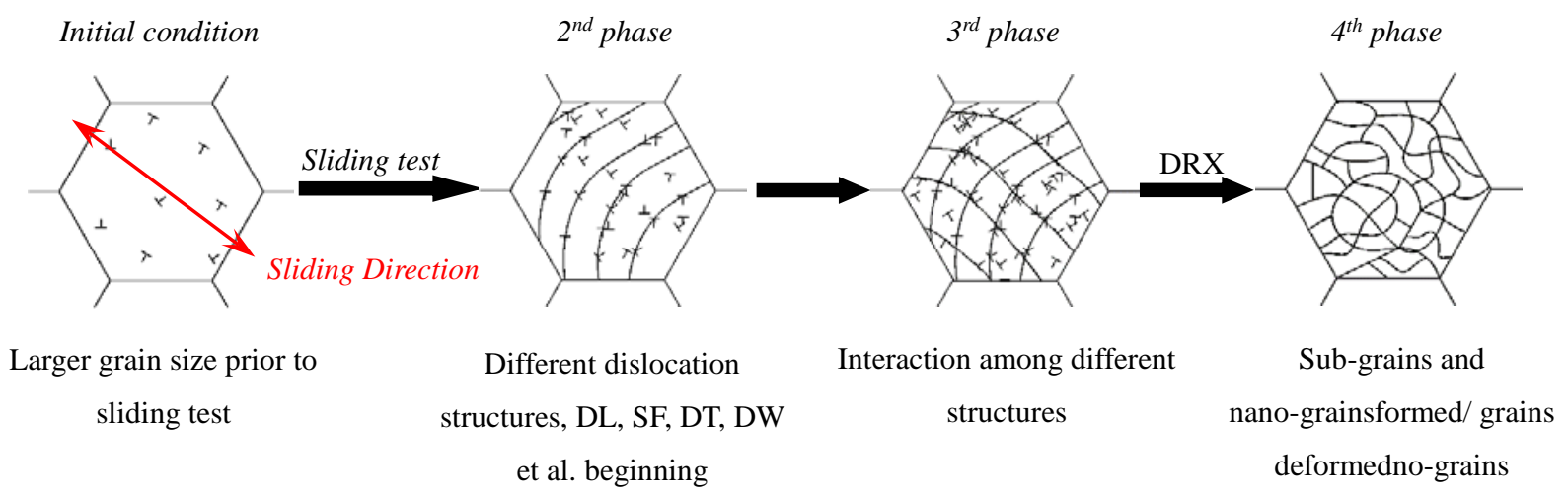

Fig.7. Schematic presentation of the grain refinement of brass during sliding test

The grain refinement mechanism in the GRZ during the sliding experiment is proposed in Fig. 7 [33]. The homogeneous dislocations in original grains are disorganized. At the beginning of the test, the friction stress causes a large number of dislocations in original grains. Subsequently, various dislocation structures such as DL, DT, DW, and SF are formed (Fig. 6(a), 6(b) and 6(c)). With the continuous sliding, the effects generated by friction stress prompts the interaction among different structures, which leads to DRX in the $3^{\text {rd }}$ phase. The DRX process causes accumulation of dislocation along the grain boundaries, eventually forming sub-grains and nano-grains. Additionally, due to the shear stress caused by friction in this phase, the dislocation movements and dislocation structures always distribute along the sliding direction, which induces the deformation of grains similar to the observation in Fig. 4(b) and Fig. 5(b).

The depth micro-hardness is obtained to investigate the mechanical properties of the third bodies. Fig. 8 shows the micro-hardness curve of the third bodies along the depth direction. The curve indicates that the depth of micro-hardness distribution is in good agreement with the depth of microstructural distribution. The micro-hardness in MML is at the highest value of about $290 \mathrm{HV}$. From the insert of Fig.8, it is obvious that the values of micro-hardness in GRZ are slightly lower than that of in MML. The micro-hardness in PDZ sharply drops along depth direction to about $60 \mathrm{HV}$ at the depth of $40 \mu \mathrm{m}$. The high level of hardness in GRZ is related to the work hardening of brass caused by the sliding test. In other words, the high dislocation density and the nano-grains are the reasons for high micro-hardness in GRZ. The effect of friction stress decreases gradually along the depth direction, which leads to the reduction trend of micro-hardness in PDZ. 


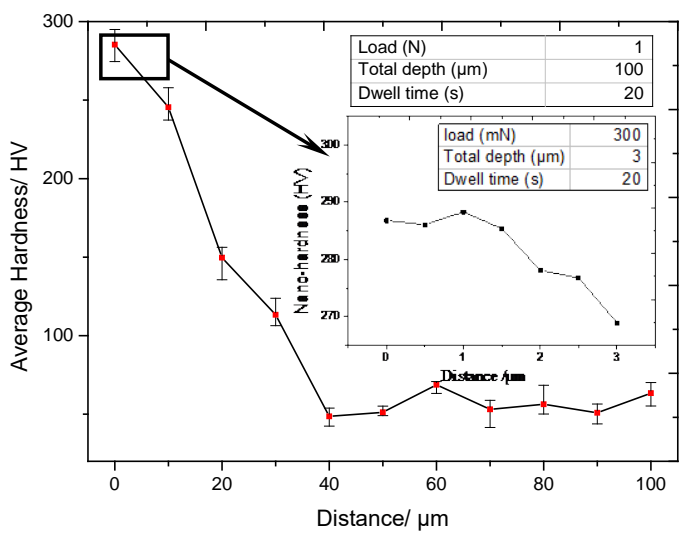

Fig. 8 The micro-hardness of the third bodies along depth direction. The insert is the nano-hardness of near-surface.

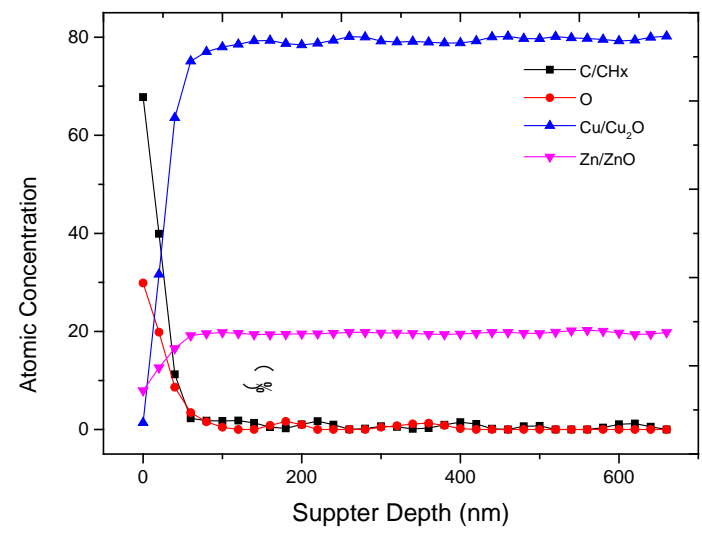

Fig. 9 The XPS profiles of the third bodies along depth direction.

No obvious grains distribution in MML is observed based on microstructure observation in Fig. 5(b). In order to study possible chemical changes of the third bodies, XPS results of the near surface layer are obtained in Fig. 9. These profiles show the atomic concentration in the third bodies along the depth direction. The sputter depth of the depth profile is $670 \mathrm{~nm}$. The contents of $\mathrm{O}$ decline from $28 \%$ to $0 \%$ on the first $100 \mathrm{~nm}$. The contents of $\mathrm{Zn} / \mathrm{ZnO}$ and $\mathrm{Cu} / \mathrm{Cu}_{2} \mathrm{O}$ increase from $7 \%$ to $21 \%$ and from $1 \%$ to $79 \%$ respectively and then remain constant. The result indicates that the main chemical compositions in $\mathrm{MML}$ are $\mathrm{Zn} / \mathrm{ZnO}$ and $\mathrm{Cu} / \mathrm{Cu}_{2} \mathrm{O}$. Oxides are the main reason for high hardness in MML (Fig. 8). The oxidation reactions occur on the surface of brass during the friction test. The accumulation of $\mathrm{ZnO}$, which is well known as a solid lubricant [34], leads to the reduction of friction for sliding. ZnO, which is as a buffer to prevent the direct contact between the friction pairs, inhibits friction and maintains the friction coefficient and wear rate with relatively low and stable in the third stage of Fig. 1(a).

3.3 Formation mechanism of the third bodies

The third bodies avoid direct contact between the friction pairs and provide a good load-carrying 
capability[34]. Moreover, they improve the stability and wear resistance of the material. Based on the above analysis, the schematic diagram of the third bodies model for brass induced by sliding test is depicted in Fig. 10. Furthermore, the macro/microscopic mechanism of the third bodies formation is proposed as follow to describe and explore the formation of the third bodies.

(I). In the early stages of the test, it is difficult for the lubricant films to completely isolate the friction pairs mainly due to relatively high viscous lubricant and the mutual sliding of asperities between friction pairs. The materials transfer from brass to pin and the adhesive wear occurs and oxidizes. The values of friction coefficient and wear rate increase sharply and oscillate at a relatively high level (Fig. 1(a)). Simultaneously, the shear stress, which is caused by plastic deformation of friction, disturbs uniform structures of original grains below the surface. A large number of dislocations are generated in the original grains.

(II). With the friction heat accumulating and continuous sliding, the lubricating film forms between the contact pairs due to the decrease of lubricant viscosity. The presence of the lubricating film relieves friction stress between the contact pairs, which provides a good condition for the formation of the third bodies. Therefore, the friction coefficient speedily drops to a low value, while the decline period of wear rate is slightly later than that of friction coefficient (Fig. 1(a)). The wear debris particles are peeled off with the sliding cycles and removed by lubricant timely, then, the fresh surface is constantly exposed. It is indicated that the MML is gradually formed on the worn surface in the later stage of the test by proceeding with oxidation reactions. Below the MML, different dislocation structures are generated in the original grains and some of them are accumulated at the grain boundaries. This mechanism of these dislocations formation was proposed by Greiner et al. [35]. Subsequently, the original grains are refined by these interactional dislocation movements and the GRZ is formed at this stage. The DRX process plays a key role in the grain refinement during the sliding period. In addition, the refined grains in GRZ show a trapezoidal distribution along the depth direction from nano-scale to micro-scale.

(III). During the sliding test, the effect of shear stress, which is generated by friction, is not sufficient to refine the grains located under GRZ, while the stress causes the shear deformation of grains in this zone (PDZ). Therefore, no obvious grains are refined in PDZ except for shear deformation of grain (Fig.4 (b) and Fig. 5(b)). In addition, a significant amount of voids are observed in PDZ (Fig. 5(a)). As a result of the experiment, severe shear plastic deformation of grain causes and accumulates dislocations, which result in voids in PDZ [36, 37]. As shown in Fig. 10, some dislocations pile up in the grains below the PDZ. 


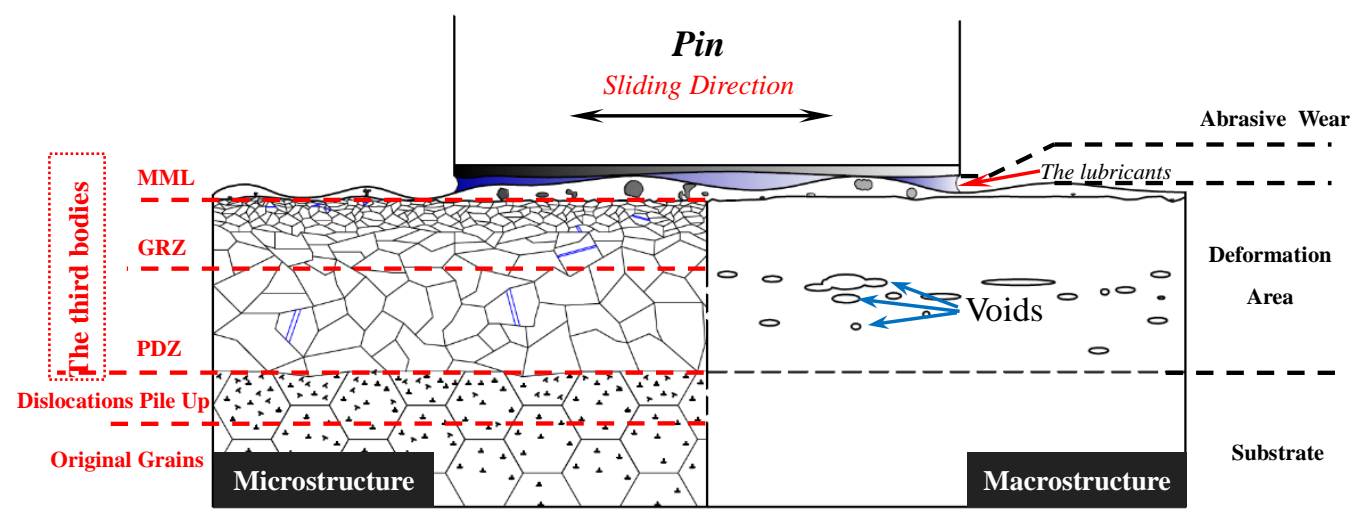

Fig. 10. Schematic diagram of the third bodies model for brass induced by sliding test.

\section{Conclusions}

(1) The running-in of brass sliding against steel under lubricated condition is studied using an on-line tribometer. Adhesive wear is the main wear mechanism in this system during the running-in stages. In the final stage, the amount of trace wear debris particles generating and peeling off reach a dynamic equilibrium with the friction coefficient and wear rate maintaining a low and stable level

(2) Based on the characterization of the microstructure and chemical composition, the third bodies (MML, GRZ, and PDZ) are formed during the sliding. ZnO, which is one of the main chemical compositions in MML, inhibits the friction effectively. The grains in GRZ are nanometer sized and the grains in PDZ show obviously shear deformation along the sliding direction.

(3) The formation mechanism of the third bodies is analyzed for the case of brass. Initially, severe friction stress leads to a large number of dislocations in the surface layer. With a continuous testing, the MML gradually is formed on the worn surface by proceeding with oxidation reactions, meanwhile, the original grains in GRZ are refined by interactional dislocation movements and DRX process. The friction stress causes grains shearing deformation in PDZ and results in voids being generated in this zone.

\section{Acknowledgements}

This work is supported by the National Natural Science Foundation of China (no. 51601021, 51671037, 51441001), the German Science Foundation DFG (Project no. DI 1494/4-1), the Natural Science Foundation of Jiangsu Higher Education Institutions (no. 17KJA460002), the China Postdoctoral Science Foundation (no. 2017M611718), the Qing Lan Project of Jiangsu Province and "Six Talent Peaks" of Jiangsu Province (no. GDZB-002). Special thanks are extended to André Blockhaus and Eberhard Nold at the Karlsruhe Institute of Technology for their assistance with tribological experiments and XPS measurements.

\section{References}


[1] J. Liu, X.-h. Wang, Q.-n. Ran, G. Zhao, X.-x. Zhu, Microstructure and properties of Cu-3Ti-1Ni alloy with aging process, Transactions of Nonferrous Metals Society of China 26(12) (2016) 3183-3188.

[2] A. Moshkovich, V. Perfilyev, T. Bendikov, I. Lapsker, H. Cohen, L. Rapoport, Structural evolution in copper layers during sliding under different lubricant conditions, Acta Materialia 58(14) (2010) 4685-4692.

[3] J.Z. Lu, H.F. Duan, K.Y. Luo, L.J. Wu, W.W. Deng, J. Cai, Tensile properties and surface nanocrystallization analyses of $\mathrm{H} 62$ brass subjected to room-temperature and warm laser shock peening, Journal of Alloys and Compounds 698 (2017) 633-642.

[4] L. Meshi, S. Samuha, S.R. Cohen, A. Laikhtman, A. Moshkovich, V. Perfilyev, I. Lapsker, L. Rapoport, Dislocation structure and hardness of surface layers under friction of copper in different lubricant conditions, Acta Materialia 59(1) (2011) 342-348.

[5] J.Z. Lu, K.Y. Luo, Y.K. Zhang, G.F. Sun, Y.Y. Gu, J.Z. Zhou, X.D. Ren, X.C. Zhang, L.F. Zhang, K.M. Chen, Grain refinement mechanism of multiple laser shock processing impacts on ANSI 304 stainless steel, Acta Materialia 58(16) (2010) 5354-5362.

[6] J. Barriga, B. Fernández-Diaz, A. Juarros, S.I.U. Ahmed, J.L. Arana, Microtribological analysis of gold and copper contacts, Tribology International 40(10-12) (2007) 1526-1530.

[7] J.Z. Lu, K.Y. Luo, Y.K. Zhang, C.Y. Cui, G.F. Sun, J.Z. Zhou, L. Zhang, J. You, K.M. Chen, J.W. Zhong, Grain refinement of LY2 aluminum alloy induced by ultra-high plastic strain during multiple laser shock processing impacts, Acta Materialia 58(11) (2010) 3984-3994.

[8] V. Perfilyev, A. Moshkovich, I. Lapsker, L. Rapoport, Friction and wear of copper samples in the steady friction state, Tribology International 43(8) (2010) 1449-1456.

[9] L.R. de Lara, R. Jagdheesh, J.L. Ocaña, Corrosion resistance of laser patterned ultrahydrophobic aluminium surface, Materials Letters 184 (2016) 100-103.

[10] J.Z. Lu, L.J. Wu, G.F. Sun, K.Y. Luo, Y.K. Zhang, J. Cai, C.Y. Cui, X.M. Luo, Microstructural response and grain refinement mechanism of commercially pure titanium subjected to multiple laser shock peening impacts, Acta Materialia 127 (2017) 252-266.

[11] P. Jha, R.K. Gautam, R. Tyagi, Friction and wear behavior of $\mathrm{Cu}-4$ wt.\%Ni-TiC composites under dry sliding conditions, Friction 5(4) (2017) 437-446.

[12] X. Li, U. Olofsson, A study on friction and wear reduction due to porosity in powder metallurgic gear materials, Tribology International 110 (2017) 86-95.

[13] Y. Kimura, Maintenance tribology: its significance and activity in Japan, Wear 207(1) (1997) 63-66.

[14] G. Schmalz, Technische Oberflächenkunde, Feingestalt und Eigenschaften von Grenzflächen technischer Körper, insbesondere der Maschinenteile, Springer-Verlag, Berlin, 1936.

[15] D.A. Rigney, L.H. Chen, M.G.S. Naylor, A.R. Rosenfield, Wear processes in sliding systems, Wear 100(1) (1984) 195-219.

[16] A. Emge, S. Karthikeyan, D.A. Rigney, The effects of sliding velocity and sliding time on nanocrystalline tribolayer development and properties in copper, Wear 267(1) (2009) 562-567.

[17] T. Feser, P. Stoyanov, F. Mohr, M. Dienwiebel, The running-in mechanisms of binary brass studied by in-situ topography measurements, Wear 303(1) (2013) 465-472. 
[18] S. Descartes, C. Desrayaud, E. Niccolini, Y. Berthier, Presence and role of the third body in a wheel-rail contact, Wear 258(7) (2005) 1081-1090.

[19] M. Scherge, A. Brink, D. Linsler, Tribofilms Forming in Oil-Lubricated Contacts, Lubricants 4(3) (2016) 27.

[20] B. Yao, Z. Han, Y.S. Li, N.R. Tao, K. Lu, Dry sliding tribological properties of nanostructured copper subjected to dynamic plastic deformation, Wear 271(9) (2011) 1609-1616.

[21] J. Dryzek, Detection of tribolayer in different metals using positron lifetime spectroscopy, Tribology International 131 (2019) 268-276.

[22] A. Singh, M. Dao, L. Lu, S. Suresh, Deformation, structural changes and damage evolution in nanotwinned copper under repeated frictional contact sliding, Acta Materialia 59(19) (2011) 7311-7324.

[23] J. Mayer, R. Engelhorn, R. Bot, T. Weirich, C. Herwartz, F. Klocke, Wear characteristics of second-phase-reinforced sol-gel corundum abrasives, Acta Materialia 54(13) (2006) 3605-3615.

[24] J.-K. Xiao, W. Zhang, C. Zhang, Microstructure evolution and tribological performance of Cu-WS2 self-lubricating composites, Wear 412-413 (2018) 109-119.

[25] Z.C. Lu, M.Q. Zeng, J.Q. Xing, M. Zhu, Improving wear performance of CuSn5Bi5 alloys through forming self-organized graphene/Bi nanocomposite tribolayer, Wear 364-365 (2016) 122-129.

[26] A. Vereschaka, V. Tabakov, S. Grigoriev, A. Aksenenko, N. Sitnikov, G. Oganyan, A. Seleznev, S. Shevchenko, Effect of adhesion and the wear-resistant layer thickness ratio on mechanical and performance properties of ZrN - (Zr,Al,Si)N coatings, Surface and Coatings Technology 357 (2019) 218-234.

[27] L. Wang, F. Duan, Nanoscale wear mechanisms of few-layer graphene sheets induced by interfacial adhesion, Tribology International 123 (2018) 266-272.

[28] C.-h. Yin, Y.-l. Liang, Y. Jiang, M. Yang, S.-l. Long, Formation of nano-laminated structures in a dry sliding wear-induced layer under different wear mechanisms of 20CrNi2Mo steel, Applied Surface Science 423 (2017) 305-313.

[29] S. Korres, M. Dienwiebel, Design and construction of a novel tribometer with online topography and wear measurement, The Review of scientific instruments 81(6) (2010) 063904.

[30] L. Liu, Z.C. Zhang, P.J. Zhan, M. Dienwiebel, Tribometer based on in-situ and radionuclide technique and its characteristic experiment study, Chinese Journal of Scientific Instrument 37(12) (2016) 2873-2879.

[31] U. Trdan, M. Skarba, J. Grum, Laser shock peening effect on the dislocation transitions and grain refinement of Al-Mg-Si alloy, Materials Characterization 97 (2014) 57-68.

[32] A. Moshkovich, V. Perfilyev, I. Lapsker, L. Rapoport, Friction, wear and plastic deformation of $\mathrm{Cu}$ and $\alpha / \beta$ brass under lubrication conditions, Wear 320 (2014) 34-40.

[33] L. Liu, Z. Zhang, M. Dienwiebel, The Running-in Tribological Behavior of Pb-Free Brass and Its Effect on Microstructural Evolution, Tribology Letters 65(4) (2017) 160.

[34] Q.Y. Zhang, S.Q. Wang, Y. Zhou, K.M. Chen, L. Wang, X.H. Cui, Artificial oxide-containing tribo-layers and their effect on wear performance of Ti-6Al-4V alloy, Tribology International 105 (2017) 334-344.

[35] C. Greiner, Z. Liu, L. Strassberger, P. Gumbsch, Sequence of Stages in the Microstructure 
Evolution in Copper under Mild Reciprocating Tribological Loading, ACS applied materials \& interfaces 8(24) (2016) 15809-19.

[36] S.H. Tang, S. Wu, M. Kobayashi, H.L. Pan, Effects of texture evolutions and point defects on ultrasonic waves under simple shear and pure shear, International Journal of Solids and Structures 44(3) (2007) 1277-1290.

[37] N.P. Suh, S. Jahanmir, I. Abrahamson, Ernest P, The delamination theory of wear, Wear 25(1) (1977) 111-124. 